\title{
UNIFIED TREATMENT OF SOME INEQUALTIES AMONG RATIOS OF MEANS
}

\author{
EMAD EL-NEWEIHI ${ }^{1}$ AND FRANK PROSCHAN ${ }^{2}$
}

\begin{abstract}
Using majorization and Schur functions, Marshall, Olkin, and Proschan obtained a result concerning monotonicity of the ratio of means. This note shows that a slight extension of their result provides a unified method for obtaining and extending inequalities between means due to Chan, Goldberg, and Gonek, as well as deriving additional inequalities of the same type.
\end{abstract}

1. Introduction. Chan, Goldberg, and Gonek [1] showed that

$$
\left[\frac{x^{p}+y^{p}}{(1-x)^{p}+(1-y)^{p}}\right]^{1 / p}<\left[\frac{x^{q}+y^{q}}{(1-x)^{q}+(1-y)^{q}}\right]^{1 / q},
$$

where $0 \leqslant x<y, x+y<1$, and $p<q$; and

$$
\left[\frac{\sum_{i=1}^{n} x_{i}^{-p}}{\sum_{i=1}^{n}\left(1-x_{i}\right)^{-p}}\right]^{-1 / p} \leqslant\left[\frac{\sum_{i=1}^{n} x_{i}^{-q}}{\sum_{i=1}^{n}\left(1-x_{i}\right)^{-q}}\right]^{-1 / q},
$$

where $0 \leqslant x_{i} \leqslant 1 / 2$ and $p>0$. Strict inequality holds in (2) unless $x_{1}=x_{2}$ $=\cdots=x_{n}$.

Earlier, Marshall, Olkin, and Proschan [2] showed

$$
\left[\frac{\sum_{i=1}^{n} \lambda_{i} a_{i}^{r}}{\sum_{i=1}^{n} \lambda_{i} b_{i}^{r}}\right]^{1 / r}
$$

is increasing in $r$, where $a_{1} \geqslant a_{2} \geqslant \cdots \geqslant a_{n}>0, b_{1} \geqslant b_{2} \geqslant \cdots \geqslant b_{n}>0, b_{1} / a_{1}$ $\leqslant b_{2} / a_{2} \leqslant \cdots \leqslant b_{n} / a_{n}$, and $\lambda_{i}>0, i=1, \ldots, n, \sum_{i=1}^{n} \lambda_{i}=1$.

Result (3) was obtained using majorization and Schur functions (for definitions see [2]).

The main purposes of this note are to show that using (3), (a) inequalities (1) and (2) can be proved in a unified way, (b) (1) and (2) can be extended, and (c) additional inequalities of a similar type can be obtained.

Received by the editors November 19, 1979.

AMS (MOS) subject classifications (1970). Primary 26A86.

Key words and phrases. Inequality among means, generalized means, majorization, Schur functions, monotonicity.

${ }^{1}$ Research sponsored by the Air Force Office of Scientific Research, AFSC, USAF, under Grant AFOSR-76-3050C.

${ }^{2}$ Research sponsored by the Air Force Office of Scientific Research, AFSC, USAF, under Grant AFOSR-78-3678. 
2. Main results. Before we state and prove the main results, we present several remarks.

REMARK 2.1. It is easy to verify that (3) holds even if certain of the $a_{i}$ 's are equal to zero.

REMARK 2.2. Careful inspection of the proof of (3) shows that in certain cases the ratio in (3) is strictly increasing in $r$.

We may now prove

TheOREM 2.3. Let $0 \leqslant x<y, x+y<1,0<\lambda<1$, and $p<q$. Then

$$
\left[\frac{\lambda x^{p}+(1-\lambda) y^{p}}{\lambda(1-x)^{p}+(1-\lambda)(1-y)^{p}}\right]^{1 / p}<\left[\frac{\lambda x^{p}+(1-\lambda) y^{q}}{\lambda(1-x)^{q}+(1-\lambda)(1-y)^{q}}\right]^{1 / q}
$$

Proof. Clearly $(1-x) x<(1-y) y$. Let $a_{1} \equiv y, a_{2} \equiv x, b_{1} \equiv 1-x$, and $b_{2} \equiv$ $1-y$. Inequality (4) follows from (3) by Remark 2.2 .

Setting $\lambda=\frac{1}{2}$ in (4) we get (1) as a special case.

The same technique yields an extension of inequality (2).

TheOREM 2.4. Let $0 \leqslant x_{i} \leqslant \frac{1}{2}, i=1, \ldots, n, p>0, \lambda_{i}>0, i=1, \ldots, n$, and $\sum_{1}^{n} \lambda_{i}=1$. Then

$$
\left[\frac{\sum_{i=1}^{n} \lambda_{i} x_{i}^{-p}}{\sum_{i=1}^{n} \lambda_{i}\left(1-x_{i}\right)^{-p}}\right]^{-1 / p}<\left[\frac{\sum_{i=1}^{n} \lambda_{i} x_{i}^{p}}{\sum_{i=1}^{n} \lambda_{i}\left(1-x_{i}\right)^{p}}\right]^{1 / p}
$$

unless $x_{1}=x_{2}=\cdots=x_{n}$.

Proof. Let $x_{[1]} \geqslant x_{[2]} \geqslant \cdots \geqslant x_{[n]}$ denote the decreasing rearrangement of $x_{1}, \ldots, x_{n}$ from now on. Let $a_{i} \equiv x_{[i]}, b_{i} \equiv\left(1-x_{[i]}\right)^{-1}, i=1, \ldots, n$. Since $-p<p$ and $\left(1-x_{[i]}\right)^{-1} x_{[j]} \leqslant\left(1-x_{[j]}\right)^{-1} x_{[i]}$ for $i<j$, we have by (3)

$$
\left[\frac{\sum_{i=1}^{n} \lambda_{i} x_{i}^{-p}}{\sum_{i=1}^{n} \lambda_{i}\left(1-x_{i}\right)^{p}}\right]^{-1 / p}<\left[\frac{\sum_{i=1}^{n} \lambda_{i} x_{i}^{p}}{\sum_{i=1}^{n} \lambda_{i}\left(1-x_{i}\right)^{-p}}\right]^{1 / p}
$$

unless $x_{1}=x_{2}=\cdots=x_{n}$ (see Remark 2.2). The desired result follows from (6).

Note that (2) is a special case of (5) by setting $\lambda_{i}=1 / n, i=1, \ldots, n$.

Finally, Theorem 2.5 below yields an inequality similar to (1) and (2). This illustrates that majorization and Schur functions can be used to generate through (3) a host of inequalities similar to (1) and (2).

THEOREM 2.5. Let $x_{i} \geqslant 0, \lambda_{i}>0, i=1, \ldots, n, \Sigma_{1}^{n} \lambda_{i}=1$, and $p<q$. Then

$$
\left[\frac{\sum_{i=1}^{n} \lambda_{i} x_{i}^{p}}{\sum_{i=1}^{n} \lambda_{i}\left(1+x_{i}\right)^{p}}\right]^{1 / p}<\left[\frac{\sum_{i=1}^{n} \lambda_{i} x_{i}^{q}}{\sum_{i=1}^{n} \lambda_{i}\left(1-x_{i}\right)^{q}}\right]^{1 / q} .
$$

Strict inequality holds in (7) unless $x_{1}=x_{2}=\cdots=x_{n}$. 
Proof. Let $a_{i} \equiv x_{[i]}$ and $b_{i} \equiv 1+x_{[i]}, i=1, \ldots, n$. Since $(1+x) / x$ is decreasing, we apply (3) to get the desired result. By Remark 2.2, strict inequality holds in (7) unless $x_{1}=x_{2}=\cdots=x_{n}$.

\section{REFERENCES}

1. F. Chan, D. Goldberg and S. Gonek, On extensions of an inequality among means, Proc. Amer. Math. Soc. 42 (1974), 202-207.

2. A. W. Marshall, I. Olkin and F. Proschan, Monotonicty of ratios of means and other applications of majorization, Inequalities (O. Shisha, Ed.), Academic Press, New York, 1967, pp. 177-190.

Department of Mathematics, University of Illinois at Chicago Circle, Chicago, Illinois 60680

Department of Statistics, Florida State University, Tallahassee, Florida 32306 